\title{
Detection of parity violation in chiral molecules by external tuning of electroweak optical activity
}

\author{
Pedro Bargueño, ${ }^{1,2, *}$ Isabel Gonzalo, ${ }^{3, \dagger}$ and Ricardo Pérez de Tudela ${ }^{1, \$}$ \\ ${ }^{1}$ Instituto de Física Fundamental (CSIC), Serrano 123, 28006 Madrid, Spain \\ ${ }^{2}$ Departamento de Química Física, Universidad de Salamanca, 37008 Salamanca, Spain \\ ${ }^{3}$ Departamento de Óptica, Universidad Complutense de Madrid, 28040 Madrid, Spain
}

(Received 10 March 2009; published 23 July 2009)

\begin{abstract}
A proposal is made to measure the parity-violating energy difference between enantiomers of chiral molecules by modifying the dynamics of the two-state system using an external chiral field, in particular, circularly polarized light. The intrinsic molecular parity-violating energy could be compensated by this external chiral field, with the subsequent change in the optical activity. From the observation of changes in the time-averaged optical activity of a sample with initial chiral purity and minimized environment effects, the value of the intrinsic parity-violating energy could be extracted. A discussion is made on the feasibility of this measurement.
\end{abstract}

DOI: 10.1103/PhysRevA.80.012110

PACS number(s): 11.30.Er, 33.80.-b

\section{INTRODUCTION}

Since the prediction and subsequent discovery of parity violation in weak interactions [1,2], the role of discrete symmetries in fundamental interactions is an intriguing field of research. The effect of electroweak interactions between electrons and nuclei mediated by the $\mathrm{Z}^{0}$ has been extensively studied and observed in atoms (see the review [3]) and only predicted in molecules, where an energy difference between the two enantiomers of chiral molecules has been estimated to be between $10^{-16}$ and $10^{-21} \mathrm{eV}$ [4-8]. In the laboratory, no conclusive energy difference has been reported in experimental spectroscopic studies reaching an energy resolution of about $10^{-15} \mathrm{eV}$ [9]. In addition, since the effect of parity violation in the optical activity (OA) was reported [10], several authors have focused their attention in the possibility of measuring the parity-violating energy difference (PVED) between enantiomers via optical rotation experiments, looking for time-dependent evolution of either chiral states [10-17] or parity states $[18,19]$. However, no experimental results have been reported up to date. The main difficulties for obtaining information about the PVED from OA experiments are the predicted very small size of the effect that can be masked by racemization processes and loss of phase coherence due to collisions with the environment.

In this work, we propose an alternative way of detecting the PVED in OA experiments. Section II gives an elementary description of the two-state system which describes chiral molecules, focusing on the role that plays the competition between parity-violating and tunneling effects in the OA. In Sec. III, we study the effect of introducing an external chiral field coupled to the dynamics of the chiral molecule on the OA, showing that it can be used to measure the PVED. Several cases are analyzed. In Sec. IV, we consider circularly polarized light (CPL) as the external chiral source and a general discussion is made. The conclusions are presented in Sec. V.

\footnotetext{
*pbdr@imaff.cfmac.csic.es

†igonzalo@fis.ucm.es

†rt@imaff.cfmac.csic.es
}

\section{CHIRAL STATES, PARITY VIOLATION, AND OPTICAL ACTIVITY}

It is well known that, in the absence of parity violation, the true stationary states of a chiral molecule are the eigenstates of parity. However, it has been shown that the effect of introducing a $P$-odd term in the Hamiltonian leads to a new set of energy eigenstates which, in certain situations, are the chiral states. For our purposes, it is enough to consider the electron-nucleon parity-violating potential as the mean contribution to this $P$-odd term. This can be included in a twostate model of a chiral molecule as a constant perturbation, $H^{P V}$, such that $H=H^{0}+H^{P V}$ is the total Hamiltonian of the system, with $H^{0}$ including only parity conserving terms. In such a situation, we can express the energy eigenstates, $| \pm\rangle$, as a linear combination of the chiral states, $|L, R\rangle$, as

$$
\left(\begin{array}{l}
|+\rangle \\
|-\rangle
\end{array}\right)=\left(\begin{array}{cc}
\cos \beta & \sin \beta \\
-\sin \beta & \cos \beta
\end{array}\right)\left(\begin{array}{l}
|L\rangle \\
|R\rangle
\end{array}\right),
$$

where $\beta$ is the mixing angle, obtained from the knowledge of the eigenvectors of this Hamiltonian $H$, getting

$$
\tan 2 \beta=\frac{2 H_{L R}}{H_{R R}-H_{L L}}=\frac{\delta}{\epsilon_{P V}},
$$

where $\epsilon_{P V} \geq 0$ means $0 \leq \beta \leq \pi / 4$ implying that $|L\rangle$ is lower or equal in energy than $|R\rangle$. The energy splitting between the two eigenstates of $H^{0}$ is $2 \delta>0$, where $\delta=\left\langle L\left|H^{0}\right| R\right\rangle$ is related to the height of the barrier of the double-well potential whose minima correspond to the respective $L$ and $R$ equilibrium conformations. The PVED is given by $\left|H_{R R}-H_{L L}\right|$ $=\left|\epsilon_{R}-\epsilon_{L}\right|=\left|2 \epsilon_{L, R}\right|$, with $\epsilon_{L}=\left\langle L\left|H^{P V}\right| L\right\rangle=-\epsilon_{R}=-\left\langle R\left|H^{P V}\right| R\right\rangle$ $\equiv \epsilon_{P V}$. One can express the eigenvalues of the system as $E_{ \pm}=E_{0} \mp \sqrt{\epsilon_{P V}^{2}+\delta^{2}}$, with $E_{0}=\left(H_{L L}+H_{R R}\right) / 2$. If $\epsilon_{P V} \rightarrow 0$, $\tan 2 \beta \rightarrow \infty$ and we recover an equal-weighted superposition of chiral states. But if $\left|\epsilon_{P V}\right| \gg \delta, \tan 2 \beta \rightarrow 0$ and the chiral states tend to be the energy eigenstates, providing a solution to Hund's paradox (the apparent stability of enantiomers despite not being in energy eigenstates) [20], as pointed out by Harris and Stodolsky [10]. 
In a general situation, if the state is $|L\rangle$ at $t=0$, the time evolution leads to the well-known oscillations between $|L\rangle$ and $|R\rangle$ states, the transition probability to the $|R\rangle$ state being

$$
P_{|L\rangle \rightarrow|R\rangle}(t)=\frac{1}{2} \frac{\delta^{2}}{\delta^{2}+\epsilon_{P V}^{2}} \sin ^{2}\left(t \sqrt{\delta^{2}+\epsilon_{P V}^{2}}\right),
$$

which tends to zero if $\left|\epsilon_{P V}\right| \gg \delta(|L\rangle$, in this case, tends to be an energy eigenstate). Note that $\hbar=1$.

As it is well known, the OA associated with the intrinsic chiral nature of some molecules leads to an opposite rotation of the plane of polarization of incident linear polarized light when it interacts with opposite enantiomers. It leads to an oscillating rotation angle of the polarization plane when the molecule is oscillating between two enantiomers. For a nonvanishing $\epsilon_{P V}$, the OA is modified (electroweak OA), so that, for a molecule $|L\rangle$ at $t=0$ and $0 \leq \beta \leq \pi / 4 \quad\left(\epsilon_{P V} \geq 0\right)$, the rotation angle is given by

$$
\Phi(t)=\Phi_{L} \frac{\epsilon_{P V}^{2}+\delta^{2} \cos \left(2 t \sqrt{\epsilon_{P V}^{2}+\delta^{2}}\right)}{\epsilon_{P V}^{2}+\delta^{2}},
$$

where $\Phi_{L}$ is the rotation angle when the molecule is in the $|L\rangle$ state. For $-\pi / 4 \leq \beta \leq 0\left(\epsilon_{P V} \leq 0\right)$, the rotation is given by

$$
\begin{aligned}
\Phi(t) & =-\Phi_{L} \frac{\epsilon_{P V}^{2}+\delta^{2} \cos \left(2 t \sqrt{\epsilon_{P V}^{2}+\delta^{2}}\right)}{\epsilon_{P V}^{2}+\delta^{2}} \\
& =\Phi_{R} \frac{\epsilon_{P V}^{2}+\delta^{2} \cos \left(2 t \sqrt{\epsilon_{P V}^{2}+\delta^{2}}\right)}{\epsilon_{P V}^{2}+\delta^{2}},
\end{aligned}
$$

where $\Phi_{R}=-\Phi_{L}$ is the rotation angle when the molecule is in the $|R\rangle$ state.

In what follows, we will focus on the time average of the relative $\mathrm{OA}$ (ROA), which for $0 \leq \beta \leq \pi / 4$ is then given by

$$
\left\langle\frac{\Phi(t)}{\Phi_{L}}\right\rangle_{t}=\frac{\epsilon_{P V}^{2}}{\epsilon_{P V}^{2}+\delta^{2}}
$$

and for $-\pi / 4 \leq \beta \leq 0$ is given by

$$
\left\langle\frac{\Phi(t)}{\Phi_{L}}\right\rangle_{t}=-\frac{\epsilon_{P V}^{2}}{\epsilon_{P V}^{2}+\delta^{2}} .
$$

There are several cases:

(i) $\left|\epsilon_{P V}\right| \ll \delta$. In this situation, the averaged ROA tends to zero since the oscillations are almost symmetric between $|L\rangle$ and $|R\rangle$ as shown in Fig. 1 (solid line). Therefore, no information about the PVED can be extracted.

(ii) $\left|\epsilon_{P V}\right| \gg \delta$. In this case in which $|L\rangle$ and $|R\rangle$ tend to be eigenstates, as mentioned above, the time-averaged value of ROA is very close to unity (as in a $|L\rangle$ state) and the extremely tiny oscillations around this value seem impossible to be observed. Let us remark that for having a timeaveraged ROA very close to unity, it is not necessary for $\epsilon_{P V}$ to be many orders of magnitude greater than $\delta$. For instance, see the case $\left|\epsilon_{P V}\right|=5 \delta$ plotted with dotted line in Fig. 1 .

(iii) If $\left|\epsilon_{P V}\right|$ is comparable to $\delta$, the competition between these magnitudes determines the behavior of the OA oscillations giving place to significative shifts in the time average of the ROA. This is illustrated in Fig. 1, where we include the particular case $\left|\epsilon_{P V}\right|=\delta$ (dashed line) in which the OA

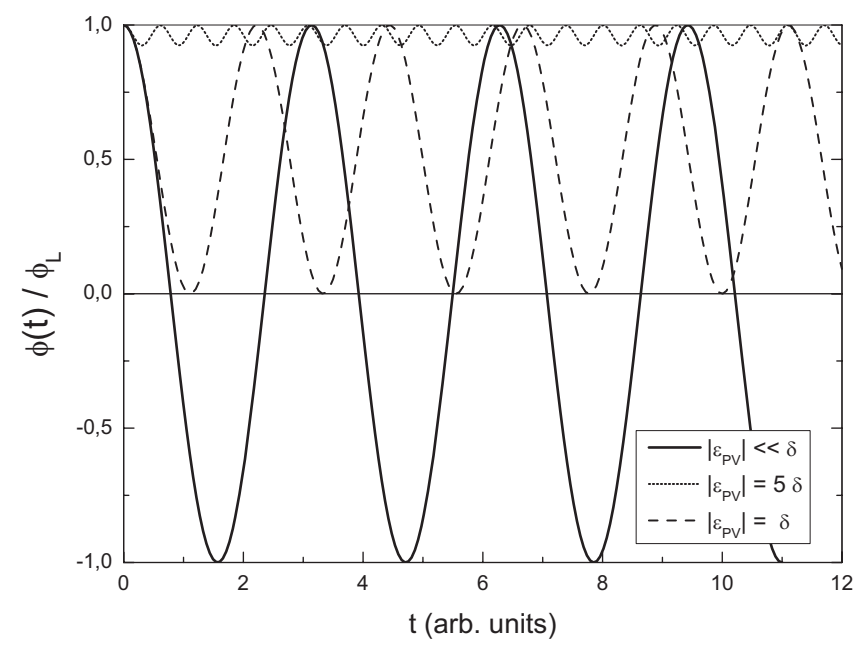

FIG. 1. Free oscillations of the relative optical activity assuming a $|L\rangle$ initial state for several cases $\left|\epsilon_{P V}\right| \ll \delta$ (solid line), $\left|\epsilon_{P V}\right|=5 \delta$ (dotted line), and $\left|\epsilon_{P V}\right|=\delta$ (dashed line).

oscillations go from $\Phi_{L}$ to zero, that is, $\left\langle\Phi(t) / \Phi_{L}\right\rangle_{t}=1 / 2$. This situation in which $\left|\epsilon_{P V}\right|$ is comparable to $\delta$ is then the most favorable to measure PVED by OA experiments, as was already noted by Harris and Stodolsky [10].

It is interesting to make some brief comments about the behavior of the time-averaged ROA under $\epsilon_{P V}$ due to $Z^{0}$ exchange in each one of the situations previously considered $\left(\left|\epsilon_{P V}\right| \ll \delta,\left|\epsilon_{P V}\right| \sim \delta\right.$, and $\left.\left|\epsilon_{P V}\right| \gg \delta\right)$. It can be observed that the time-averaged ROA due to $Z^{0}$ exchange tends to unity as long as $\epsilon_{P V}$ increases, thus showing the expected behavior since when parity violation dominates the dynamics, no oscillations of the OA are expected. The main difference between the three considered situations is then that the approach to unity is faster as long as the value of $\epsilon_{P V} / \delta$ is bigger.

Up to date, there have been several proposals to measure the electroweak OA. Harris and Stodolsky [10] proposed to prepare one of the chiral states and to measure the oscillations of the OA around a nonzero value. Quack $[18,19]$ considered to measure oscillations of $\mathrm{OA}$ from an initially prepared $|+\rangle$ or $|-\rangle$ state. Harris [16] and MacDermott and Hegstrom [17] pointed out the difficulties of these proposals, suggesting to apply an external electric field to enhance the OA. The most recent work in this line [17] focuses on the measurement of PVED by means of separating the states $|+\rangle$ and $|-\rangle$ using an inhomogeneous electric field. The authors calculate the OA of the separated states, which is given by $\Phi_{ \pm} \sim \pm \Phi_{L}\left(\epsilon_{P V} / \delta\right)$, and show that it should be measurable within the capabilities of state-of-the-art polarimeters.

In the next section, we describe an alternative method that would allow us to measure the PVED by means of an external chiral field (in particular CPL). We will see that the internal dynamics driven by parity violation and tunneling can be controlled with this field.

\section{EXTERNAL CONTROL OF OPTICAL ACTIVITY AND CONSEQUENCES FOR THE DETECTION OF THE PVED}

Our proposal lies in the apparent trivial fact that the intrinsic molecular PVED could be compensated by an exter- 
nal chiral perturbation. In this case, the electroweak OA would change accordingly. We consider now that the zerothorder Hamiltonian includes both $H^{0}$ and $H^{P V}$. If a chiral perturbation, $H^{\prime}$, satisfying $\epsilon^{\prime}=\left\langle L\left|H^{\prime}\right| L\right\rangle=-\left\langle R\left|H^{\prime}\right| R\right\rangle$, is applied, the new mixing angle $\beta^{\prime}$ is obtained by an analogous method to that followed to get Eq. (2), so it is given by

$$
\tan 2 \beta^{\prime}=\frac{\delta}{\epsilon_{P V}+\epsilon^{\prime}},
$$

and Eq. (4) is then trivially modified as

$$
\Phi(t)=\Phi_{L} \frac{\epsilon^{2}+\delta^{2} \cos \left(2 t \sqrt{\epsilon^{2}+\delta^{2}}\right)}{\epsilon^{2}+\delta^{2}},
$$

where $\epsilon \equiv \epsilon_{P V}+\epsilon^{\prime}$. The time-averaged ROA expressions are then modified as

$$
\left\langle\frac{\Phi(t)}{\Phi_{L}}\right\rangle_{t}=\frac{\epsilon^{2}}{\epsilon^{2}+\delta^{2}},
$$

for $0 \leq \beta^{\prime} \leq \pi / 4$, and

$$
\left\langle\frac{\Phi(t)}{\Phi_{L}}\right\rangle_{t}=-\frac{\epsilon^{2}}{\epsilon^{2}+\delta^{2}},
$$

for $-\pi / 4 \leq \beta^{\prime} \leq 0$.

Let us consider the free time evolution of a molecule with $\epsilon_{P V} \neq 0$ in a well defined $|L\rangle$ state at $t=0$. In absence of the external chiral field, its time-averaged ROA is given by $\epsilon_{P V}^{2} /\left(\epsilon_{P V}^{2}+\delta^{2}\right)$ as shown in Eq. (6). By applying now a chiral field of appropriate handedness and intensity, the timeaveraged ROA given by Eq. (10) becomes zero when $\epsilon^{\prime}=-\epsilon_{P V}$ (that is, $\epsilon=0$ ), which permits to get a value of $\epsilon_{P V}$ if the external perturbation $\epsilon^{\prime}$ is known. If the intensity of the external field is further increased, the averaged ROA reverses its sign being described by Eq. (11). An additional increase of the external field up to $\epsilon^{\prime}=-2 \epsilon_{P V}$ leads to a time-averaged ROA given by $-\epsilon_{P V}^{2} /\left(\epsilon_{P V}^{2}+\delta^{2}\right)$, which is opposite to the initial one without any external chiral field. This behavior is shown in Fig. 2 for several cases. Let us now analyze how significant the changes are in the OA when $\epsilon^{\prime}$ is of the order of $\epsilon_{P V}$ in the three cases mentioned in the preceding section.

(i) For $\left|\epsilon_{P V}\right| \ll \delta$, the dynamics is determined by tunneling but not by PVED. Since $\epsilon^{\prime}$ is as small as PVED, there is no significant change in the OA of the molecule, as in the situation depicted in Fig. 1 with solid line.

(ii) For $\left|\epsilon_{P V}\right| \gg \delta$ (dotted line in Fig. 1), $\delta$ is so small in this case that although $\epsilon_{P V}$ could be compensated with $\epsilon^{\prime}$, no changes in the time-averaged ROA would be observed in general due to very long tunneling time (too large for laboratory times or for keeping coherence). Among all the molecules reported in the review made by Quack [21], the most favorable molecule belonging to this case is $\mathrm{D}_{2} \mathrm{Te}_{2}$ whose calculated tunneling time of about $16 \mathrm{~s}$ is probably still too large for keeping molecular coherence as will be commented below.

(iii) Finally, in the most favorable case in which $\left|\epsilon_{P V}\right|$ $\sim \delta$, we must distinguish three different situations:

(a) If $\left|\epsilon_{P V}\right|=\delta$, a measure of the time-averaged ROA (here is $1 / 2$ ) would give already the value of $\epsilon_{P V}$ if $\delta$ is known. If $\delta$ is not known, the external chiral field can be applied in

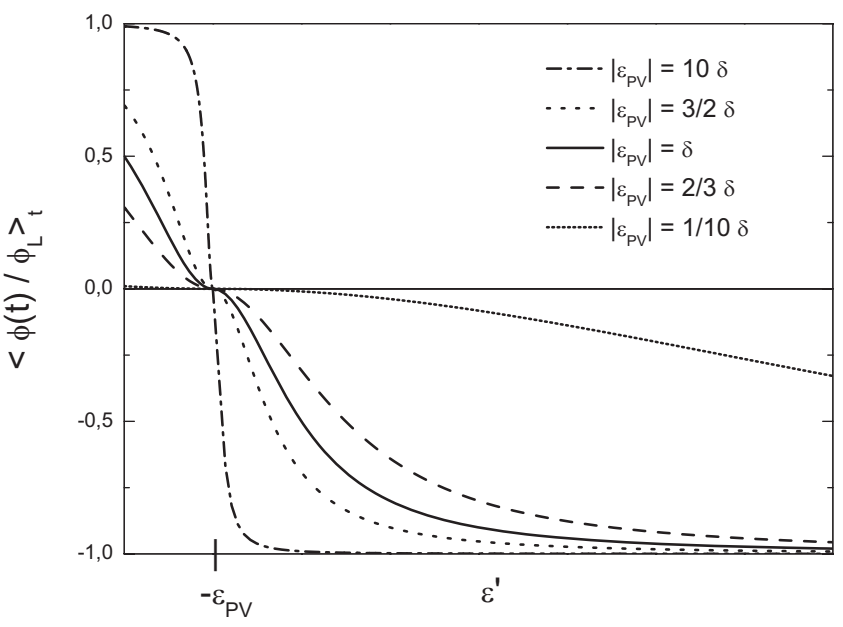

FIG. 2. Time average of the relative optical activity vs the perturbation energy $\left(\epsilon^{\prime}\right)$ supplied by the external chiral field for several cases. Note that there is a change of sign when $\epsilon^{\prime}=-\epsilon_{P V}$ (see the text).

order to obtain zero or a change of sign in the averaged ROA, which permits a measure of $\epsilon_{P V}$ as explained above. Among the molecules reported by Quack [21], the only one satisfying this constraint is $\mathrm{T}_{2} \mathrm{~S}_{2}$. However, its calculated tunneling time of about $16 \mathrm{~s}$ seems too large for keeping molecular coherence.

(b) If $\left|\epsilon_{P V}\right|<\delta$, these magnitudes must be related by a factor significantly lower than 10 to obtain an appreciable change in the averaged ROA when it goes to zero or when it reverses its sign (a factor of 10 gives an averaged ROA of about $10^{-2}$, which is too close to zero for our purposes). Among the molecules reported by Quack [21], the calculated values of the molecule $\mathrm{D}_{2} \mathrm{Se}_{2}$ verify $\left|\epsilon_{P V}\right|=(2 / 3) \delta$, the tunneling time being of $55 \mathrm{~ms}$. In this case, the averaged ROA is about 0.3 and a change to zero or to -0.3 by means of the external field could be appreciated. In the same review [21], the molecule $\mathrm{H}_{2} \mathrm{Te}_{2}$ is reported with tunneling time of 0.55 ms (more favorable for keeping coherence), however the calculated values verify $\left|\epsilon_{P V}\right|=\delta / 10$ and the changes would be then difficult to be appreciable as was said.

(c) In the case $\left|\epsilon_{P V}\right|>\delta$, we note that if these magnitudes are related by a factor of about 10 , the averaged ROA is already close to unity, which seems more favorable than other cases to detect a change to zero of the averaged ROA. To distinguish experimentally this last situation from that in which there is no $\epsilon_{P V}$ and the tunneling time is longer than laboratory times $\left[\Phi(t \rightarrow \infty)=\Phi_{L}\right]$, the external chiral field seems to be useful in the following way. Large tunneling times imply high-energy barriers, so an external chiral field that produces a small contribution $\epsilon^{\prime}$ of the order of $\epsilon_{P V}$ could not induce an inversion of the molecule through the high barrier. However, if such a field is able to induce a zero averaged ROA, we can conclude that the external field is able to compensated the $\epsilon_{P V}$ value of an oscillating molecule. Among the molecules reported by Quack [21], there is no one in this case (c) with $\left|\epsilon_{P V}\right|$ and $\delta$ being comparable. 


\section{CIRCULARLY POLARIZED LIGHT AS THE EXTERNAL CHIRAL FIELD AND GENERAL DISCUSSION}

The conclusions extracted up to this point have been derived from the basic assumption that the applied external field is chiral. It is well known that a static uniform electric or magnetic field does not represent a truly chiral influence according to the generally accepted definition: true chirality is shown by systems existing in two distinct enantiomeric states that are interconverted by space inversion, but not by time reversal combined with any proper spatial rotation [22]. Nevertheless, if the electric or magnetic fields are nonuniform, true chirality may exist for certain configurations (see the extensive review by Ávalos and co-workers [23] about chiral influences and absolute asymmetric synthesis).

The true chirality of polarized photons can be easily demonstrated by considering the effects of parity and time reversal on the system in question. The photons in a beam of CPL radiation propagating as a plane wave are in spin angularmomentum states with a spin quantum number $s=1$ and quantum numbers $m_{s}= \pm 1$. A circularly polarized photon shows true chirality since parity interconverts the right and left circularly polarized forms, but time reversal does not. Then, our general statements about how to control the electroweak OA do apply using CPL as external chiral field.

The Hamiltonian, $H^{\prime}$, for the interaction of CPL with a molecule reads

$$
H^{\prime}=-\mu \cdot \mathbf{E}^{ \pm}(t)-\mathbf{m} \cdot \mathbf{B}^{ \pm}(t),
$$

where $\mu$ and $\mathbf{m}$ are the electric and magnetic-dipole moment operators, $\mathbf{E}^{ \pm}(t)$ and $\mathbf{B}^{ \pm}(t)$ are the circularly polarized electric and magnetic fields, and the signs \pm standing for the handedness of polarization. In the preceding section, the effect of $H^{\prime}$ on the enantiomers of a chiral molecules was denoted by $\epsilon^{\prime}=\left\langle L\left|H^{\prime}\right| L\right\rangle=-\left\langle R\left|H^{\prime}\right| R\right\rangle$. The energy difference, $2 \epsilon^{\prime}$, between the two enantiomers due to the interaction with CPL was calculated by Shao and Hänggi [24]. The major contribution of this energy is given by

$$
2 \epsilon^{\prime}= \pm \frac{16}{3} E B \omega \frac{R_{n 0}}{\omega_{n 0}^{2}-\omega^{2}},
$$

where $\omega_{n 0}$ is the resonance frequency nearest the incident frequency $\omega, R_{n 0}=\operatorname{Im}\left(\mu_{\mathrm{n} 0} \cdot \mathbf{m}_{\mathrm{n} 0}\right)$ is the rotational strength, and a time average is made since the period of the electromagnetic radiation is much shorter than the molecular time oscillations.

In order to estimate the value of $\epsilon^{\prime}$, we approximate the electric-dipole moment by $e a_{0}\left(a_{0}\right.$ is the Bohr radius) and the magnetic-dipole moment by the Bohr magneton $(e / 2 m c)$ which leads to $R_{n 0} \sim 10^{-18} \mathrm{Cm} / \mathrm{Ts}$. If we consider $B=E / c$, $\omega^{2} /\left(\omega_{n 0}^{2}-\omega^{2}\right)$ of the order of unity [25], and $\omega \approx 3$ $\times 10^{15} \mathrm{rad} \mathrm{s}^{-1}$ (red light), we obtain

$$
\epsilon^{\prime} \sim \pm 3 \times 10^{-24} E^{2} \mathrm{eV} .
$$

This energy must be able to compensate the parity-violating energy $\epsilon_{P V}$ of the order between $10^{-16}$ and $10^{-18} \mathrm{eV}$ (see Table II of Ref. [21].) for most of the chiral molecules. From the condition $\epsilon^{\prime}=\epsilon_{P V}$, we obtain that the electric field of the CPL must be in the range

$$
200 \leq E \leq 2000 \mathrm{~V} \mathrm{~m}^{-1},
$$

which is easily achieved in the laboratory by usual lasers with powers in the range of from some $\mathrm{mW} \mathrm{cm}^{-2}$ to about $1 \mathrm{~W} \mathrm{~cm}^{-2}$.

Then, with this type of external field, it would be possible to control the time-averaged optical activity of the molecular sample provided each molecule keeps its coherence during its free time evolution.

Keeping coherence is one of the major experimental difficulties, as commented in several works (e.g., [17]). Since we try to measure the time average of the optical rotation, related to the time average of the oscillations, we do not require neither the initial time $t=0$ of their free oscillations be the same nor the ensemble of molecules oscillate together coherently. However, it is essential that each molecule preserves its individual coherence, evolving as Eq. (9). For this to occur, the time between collisions must be much longer than the molecular tunneling time. According to the discussion from [17], if we consider typical molecular beams (velocity of $\sim 100 \mathrm{~m} / \mathrm{s}$ and path length of about $1 \mathrm{~m}$ ), we would require a tunneling time of $\tau<10^{-2} \mathrm{~s}$. Advances in molecular beams allow the molecules to be highly dilute at a few degrees Kelvin, with extremely low velocities, so still minimizing collisional effects. Moreover, it is possible to include helium droplets in molecular beams [26] which have the ability to capture molecules inside them [27]. This feature offers promising possibilities to improve the degree of isolation of the molecules. The superfluid nature of bosonic helium droplets below $2.17 \mathrm{~K}$ makes it possible for molecules placed inside them to evolve with zero friction, as in vacuum. In this scenario, the collision rate can be drastically reduced to almost zero and all the spurious thermal effects vanish due to the evaporation of helium. In such conditions, molecular coherence could be maintained for tunneling times much longer than $10^{-2} \mathrm{~s}$.

Another aspect to be taken into account is that electromagnetic fields with very specific conditions could destroy tunneling, producing complete localization of the enantiomers $[24,28]$. Hence, the CPL applied to the molecular sample, as well as the linear polarized field whose optical rotation would be measured, must be far from these specific conditions.

Apart from these problems, the molecular sample must have initial chiral purity, e.g., $L$. In this sense, laser technology has prompted a considerable number of works dealing with the control of molecular handedness with the aim of obtaining chiral purity from racemic mixtures in molecular beams. Let us mention, for example, the proposal of Kucirka and Shekhtmann [29] using lasers with CPL in molecular beams, the works of Hoki et al. using linearly polarized femtosecond laser pulses in an oriented sample [30] or using three polarization components of electric fields in a randomly oriented sample in another laser scenario [31], and the work of Ma and Salam [32] using circularly polarized pulsed lasers. 


\section{CONCLUSIONS}

A proposal is made to measure the parity-violating energy difference, $2 \epsilon_{P V}$, between the enantiomers of a chiral molecule by measuring changes (induced by an external chiral field) in the time-averaged relative optical activity (ROA) of a molecular sample prepared with chiral purity at initial time. An external chiral field such as CPL generated in available lasers (lying in the range of $\mathrm{mW} \mathrm{cm}^{-2}-\mathrm{W} \mathrm{cm}^{-2}$ ) can induce an energy, $\epsilon^{\prime}$, of the order of the parity-violating energy in chiral molecules, $\epsilon_{P V}$. By varying the intensity of this field, the time-averaged ROA can be controlled so that when the external field compensates $\epsilon_{P V}$, the time-averaged ROA becomes zero. The external field can also induce a change of sign in the time-averaged ROA. It would allow us to detect the parity-violating energy and even to measure it if $\epsilon^{\prime}$ is known.

Several situations depending on the relative values of the parity-violating energy difference, $2 \epsilon_{P V}$, and the splitting, $2 \delta$, which determines tunneling times through the barrier, are discussed. The most favorable cases arise when these values are comparable (since the time-averaged ROA is then appreciable) and the tunneling time is short enough to preserve molecular coherence. One of the most promising candidates is $\mathrm{D}_{2} \mathrm{Se}_{2}$ [21], with theoretical values satisfying $\left|\epsilon_{P V}\right|$ $=(2 / 3) \delta$ with tunneling time of $55 \mathrm{~ms}$.

The observation of a time-averaged ROA does not require coherence in the ensemble of the molecules provided each molecule preserves its individual coherence in the oscillations at least during the experimental time. Advances in molecular beams open possibilities to minimize environment effects (and then decoherence) even by isolating chiral molecules inside helium clusters.

\section{ACKNOWLEDGMENTS}

This was funded by the MEC (Spain) under Projects No. CTQ2005-09185-C02-02, No. FIS2004-02461, and No. FIS2007-65382 and supported by Grants No. BES-200611976 (P.B.) and No. BES-2006-7454 (R.P.deT.).
[1] T. D. Lee and C. N. Yang, Phys. Rev. 104, 254 (1956).

[2] C. S. Wu et al., Phys. Rev. 105, 1413 (1957).

[3] A. M. Bouchiat and C. C. Bouchiat, Rep. Prog. Phys. 60, 1351 (1997).

[4] V. S. Letokhov, Phys. Lett. A 53, 275 (1975).

[5] R. A. Hegstrom, D. W. Rein, and P. G. H. Sandars, J. Chem. Phys. 73, 2329 (1980).

[6] R. Zanasi, P. Lazzeretti, A. Ligabue, and A. Soncini, Phys. Rev. E 59, 3382 (1999).

[7] J. K. Laerdahl, P. Schwerdtfeger, and H. M. Quiney, Phys. Rev. Lett. 84, 3811 (2000).

[8] P. Soulard et al., Phys. Chem. Chem. Phys. 8, 79 (2006).

[9] J. Crassous et al., Org. Biomol. Chem. 3, 2218 (2005).

[10] R. A. Harris and L. Stodolsky, Phys. Lett. 78B, 313 (1978).

[11] R. A. Harris and R. Silbey, J. Chem. Phys. 78, 7330 (1983).

[12] R. A. Harris, Chem. Phys. Lett. 223, 250 (1994).

[13] R. A. Harris, Y. Shi, and J. A. Cina, J. Chem. Phys. 101, 3459 (1994).

[14] R. Silbey and R. A. Harris, J. Chem. Phys. 93, 7062 (1989).

[15] R. A. Harris and L. Stodolsky, J. Chem. Phys. 74, 2145 (1981).

[16] R. A. Harris, Chem. Phys. Lett. 365, 343 (2002).

[17] A. J. MacDermott and R. A. Hegstrom, Chem. Phys. 305, 55
(2004).

[18] M. Quack, Chem. Phys. Lett. 132, 147 (1986).

[19] M. Quack, Angew. Chem., Int. Ed. Engl. 28, 571 (1989).

[20] F. Hund, Z. Phys. 43, 805 (1927).

[21] M. Quack, Annu. Rev. Phys. Chem. 59, 741 (2008).

[22] L. D. Barron, Mol. Phys. 43, 1395 (1981).

[23] M. Avalos et al., Chem. Rev. 98, 2391 (1998).

[24] J. Shao and P. Hänggi, J. Chem. Phys. 107, 9935 (1997).

[25] L. D. Barron, Molecular Light Scattering and Optical Activity, 2nd ed. (Cambridge University Press, London, 2004).

[26] W. Schollkopf and J. P. Toennies, Science 266, 1345 (1994).

[27] S. Goyal, D. L. Schutt, and G. Scoles, Phys. Rev. Lett. 69, 933 (1992).

[28] F. Grossmann, T. Dittrich, P. Jung, and P. Hänggi, Phys. Rev. Lett. 67, 516 (1991).

[29] J. Kucirka and A. G. Shekhtmann, Phys. Lett. A 221, 273 (1996).

[30] K. Hoki, L. González, and Y. Fujimura, J. Chem. Phys. 116, 2433 (2002).

[31] K. Hoki, L. González, and Y. Fujimura, J. Chem. Phys. 116, 8799 (2002).

[32] Y. Ma and A. Salam, Chem. Phys. 324, 367 (2006). 